\title{
Participación de los trabajadores en las utilidades o resultados de las empresas en América Latina
}

\section{Andrés E. Marinakis}

Especialista en Políticas del Mercado de Trabajo e Instituciones Laborales Equipo Técnico Multidisciplinario para Argentina, Brasil, Chile, Paraguay y Uruguay con sede en Santiago de Chile. Organización Internacional del Trabajo (оIт)
Este artículo analiza los sistemas de participación de los trabajadores en las utilidades o resultados de las empresas que se aplican en América Latina, así como su capacidad para responder a los requerimientos del contexto económico en que se desempeñan las firmas. Tras una parte introductoria (sección I), se presentan brevemente los sistemas de remuneración variable y se describen las características principales de los sistemas de participación en cuatro países de América Latina: Chile, México, Perú y Venezuela (sección II). Seguidamente, se comparan las características de estos sistemas entre ellos y con las del sistema adoptado en Brasil a partir de 1994 (sección III). Se evalúa luego la aplicación de estos instrumentos de participación. Con tal objeto, se presenta información relativa al sistema de participación en las utilidades de México (sección IV), y a partir de información aún bastante fragmentaria, se discuten algunos aspectos que parecen importantes para hacer una evaluación preliminar del sistema brasileño (sección V). A modo de conclusión, se examina la oportunidad de aplicación de estos instrumentos de participación en el nuevo contexto económico-laboral (sección VI). 


\section{I}

\section{Introducción}

La mayor parte de los países latinoamericanos en donde existe legislación relativa a la participación financiera de los trabajadores en las empresas cuenta con sistemas de participación en las utilidades (llamadas en algunos casos beneficios, ganancias o también lucros). En principio, los programas de participación en las utilidades consisten en pagos adicionales realizados a los trabajadores en función del logro de un cierto beneficio por parte de sus empresas. Por lo general, en estos países los sistemas de participación fueron introducidos a través de legislación de aplicación obligatoria. En ella se establece el porcentaje de participación, la definición de beneficio que se debe utilizar, los trabajadores comprendidos y las excepciones, y a veces la forma en que el monto establecido debe ser distribuido entre los trabajadores. Por lo tanto, en esencia se trata de un pago variable que procura ligar una parte de la remuneración total de los trabajadores al éxito de sus empresas medido por las ganancias de éstas.

En su origen, a principios de este siglo, los sistemas de participación en las utilidades fueron instrumentados en forma voluntaria en empresas que procuraban establecer buenas relaciones laborales y querían compartir parte de los frutos de su éxito con los trabajadores que las conformaban (Perry y Kegley, 1990). Detrás de esta visión había una moderada intención redistributiva. Más adelante, algunos países quisieron establecer este principio en forma obligatoria, extendiendo el beneficio a la mayoría de los trabajadores. En un contexto de pleno empleo y crecimiento sostenido, esta política distributiva parecía estar de acuerdo con el contexto económico-laboral.

En los últimos años la situación ha sufrido sin duda cambios importantes. El mercado laboral ya no se caracteriza por relaciones contractuales indefinidas, sino que proliferan formas de contratación precarias, y las empresas parecen haber entrado en un proceso de reestructuración sin fin impulsado por la necesidad de mantener su competitividad, ahora a nivel internacional. Cabe preguntarse, entonces, si en este nuevo contexto la participación de los trabajadores en las ganancias continúa siendo un instrumento apropiado tal como fuera formulado.

Este mecanismo no es novedoso a nivel regional. En Chile, México, Perú y Venezuela, entre otros países, existen distintos instrumentos que regulan esta participación, con un común objetivo original, el de expresar el derecho de los trabajadores a participar en el resultado financiero de las empresas y una evidente intención redistributiva.

Apartándose de esta práctica predominante en América Latina, recientemente Brasil ha adoptado un enfoque diferente de la participación de los trabajadores. La Medida Provisional 794, introducida a fines de 1994, establece la participación de los trabajadores en los lucros o resultados de las empresas. ${ }^{1}$ Esta MP reglamenta por primera vez una disposición constitucional que existía desde 1946 y que fue ratificada en las sucesivas constituciones brasileñas. Sus términos son bastante flexibles: los actores sociales pueden definir los criterios de participación mediante la negociación, y pueden optar por un sistema ligado al lucro de la empresa, a resultados específicos, o bien a indicadores mixtos. Dado que este instrumento legal está en vigor desde hace tres años, existe alguna experiencia en su aplicación que puede ser útil para evaluar las bondades de este nuevo enfoque.
Una versión preliminar de este documento fue presentada en el seminario tripartito sobre "Experiencias de participación de los trabajadores en los lucros o resultados de las empresas", realizado en São Paulo en agosto de 1997. La responsabilidad de las opiniones expresadas en este artículo incumbe exclusivamente a su autor y su publicación no implica que la ort las sancione.

\footnotetext{
1 De acuerdo con lo establecido en la Constitución brasileña, en caso de relevancia y urgencia el Presidente de la República puede adoptar medidas provisionales, con fuerza de ley, debiendo someterlas de inmediato al Congreso Nacional. El plazo de su vigencia es de treinta días, y caducan en el caso de no ser convertidas en ley. Para evitar interrupciones en su vigencia, las MP pueden ser reeditadas con un nuevo número, tal cual ha sucedido con la Medida Provisional en cuestión hasta el momento.
} 


\section{II}

\section{Sistemas de remuneración variable}

Si bien la participación en los lucros y la participación en los resultados pueden ser consideradas parte de sistemas de remuneración variable, más allá de las diferencias en su implementación, ambas tienen potencialidades y objetivos bien diferentes que conviene aclarar.

Los sistemas de participación en las utilidades pagadera en dinero (como los de todos los países latinoamericanos) son fundamentalmente sistemas de dis- tribución del ingreso que, en el mejor de los casos, aumentan en cierta medida la identificación del trabajador con la empresa. ${ }^{2}$ Sin embargo, en la mayoría de los casos no pasan de ser un "costo adicional" al proceso de producción, aunque de carácter variable. Esto último, evidentemente, representa una ventaja frente al resto de los costos laborales, ya que en momentos de crisis que hagan caer las utilidades de la empresa este componente se ajusta a la baja en forma automática.

\section{Recuadro 1}

PRINCIPALES DIFERENCIAS ENTRE LA PARTICIPACIÓN EN LOS LUCROS Y LA PARTICIPACIÓN EN LOS RESULTADOS

\section{Participación en los lucros (utilidades)}

Objetivo

Factor que se remunera

Unidad que se remunera

Frecuencia de la

remuneración

Disponibilidad de la retribución

Principal ventaja

Principal desventaja

Fuente: Marinakis (1997).
Ligar las remuneraciones al éxito de la empresa

Promover la identificación de los trabajadores con los objetivos de la empresa (aumentar las utilidades)

Lucros/utilidades

Empresa como un todo o unidades fabriles

Anual o semestral

Inmediata (pago en efectivo, sin beneficio fiscal para el trabajador)

Mediata (pago diferido, con incentivo fiscal para el trabajador)

En períodos de lucro, da una parte a los trabajadores. En período recesivo, el costo laboral se reduce en forma automática, aliviando el ajuste

Factores importantes no están bajo el control de los trabajadores (decisiones de la dirección o factores macroeconómicos)

\section{Participación en los resultados}

Puede ser diseñada para:

Estimular el esfuerzo individual

Mejorar la calidad del producto

Economizar en el uso de recursos

Estimular la cooperación y el trabajo en grupo

Productividad (medida a partir de uno o varios indicadores)

Individuos o grupos de trabajo

Mensual, trimestral, semestral

Inmediata (pago en efectivo, sin beneficio fiscal para el trabajador)

Los trabajadores tienen mayor influencia y control sobre el resultado que en el caso de planes de participación en los lucros

A veces es difícil determinar y medir los indicadores más adecuados. Se debe evitar que el incentivo tenga efectos indeseados sobre otros aspectos.

\footnotetext{
${ }^{2}$ No analizaremos aquí los sistemas de participación con pagos diferidos en el tiempo, los que pueden tener un impacto positivo sobre
}

la tasa de ahorro interno. La prevalencia de niveles salariales muy bajos en los países analizados haría sumamente difícil su aplicación. 
Esta flexibilidad de los costos laborales constituye el argumento teórico central en el planteamiento de Martin Weitzman. Desde su punto de vista, vincular una parte significativa de las remuneraciones totales a las utilidades de las empresas puede llevar a un aumento del empleo y a una reducción de las presiones inflacionarias de los salarios (Weitzman, 1984). En lo que hace al empleo, ligar una parte importante de las remuneraciones al desempeño financiero de las empresas puede estimular nuevas contrataciones, ya que reduce tanto el riesgo empresarial como el costo de las nuevas contrataciones que participarán del monto total de utilidades prefijado. En lo que hace a la inflación, al vincular una parte de los aumentos de las remuneraciones a las ganancias se restringen las causas salariales de la inflación.

Los sistemas de participación en los resultados, por su parte, pueden introducir o estimular cambios en los comportamientos de los trabajadores y en su utilización de los factores de producción, con posibles beneficios tanto para la empresa como para el personal. En conversaciones entre la dirección y los trabajadores se establecen metas medibles para variables que están bajo el control de estos últimos y una escala de premios de acuerdo con su consecución. Para potenciar la motivación, los pagos se deben realizar con la mayor frecuencia posible, para relacionarlos así con la mejora en el desempeño. Los programas pueden estar orientados a estimular el esfuerzo individual (aumentando la productividad en sentido estricto), mejorar la calidad del producto, economizar en el uso de recursos, disminuir el desperdicio y el tiempo ocioso, reducir los accidentes de trabajo, etc. Incluso se puede incentivar más de un aspecto a la vez.

La característica de los sistemas de participación en las utilidades que mejor encaja en el contexto económico actual es su carácter variable. Los pagos por participación en las ganancias forman parte de la remuneración total de los trabajadores, pero a diferencia de lo que sucede con el salario base u otras bonificaciones que se pagan en forma regular (decimotercer salario, vacaciones) o que dependen de las características individuales (subsidio familiar, antigüedad), sólo se materializan si las ganancias efectivamente se concretan. En caso contrario, sencillamente no se realiza pago alguno por este concepto. Por lo tanto, este componente variable de la remuneración total es un instrumento de ajuste automático de los costos laborales, que puede dar flexibilidad a la empresa ante fluctuaciones en la demanda de su producto y contribuir a evitar despidos innecesarios. ${ }^{3}$

Varios estudios han procurado establecer la relación entre la participación en las utilidades y la productividad. Una revisión de las publicaciones sobre el tema estableció que existe una relación positiva, aunque moderada (Weitzman y Kruse, 1990), pero que el efecto de esa participación sobre la productividad se daría al momento de iniciar los programas, por lo cual se trataría de un efecto no repetitivo (Card, 1990).

Sin embargo, la participación de los trabajadores en las utilidades no parece influir significativamente en su motivación. Esto limitaría la utilidad del instrumento en momentos en que los procesos de reestructuración organizacional en el ámbito de las empresas requieren de la colaboración y participación activa de los trabajadores. En este sentido, la participación de los trabajadores en los resultados aparece como un instrumento más adecuado, ya que en este caso el pago variable está ligado al logro de metas específicas de interés para la empresa, previamente acordadas entre los trabajadores y la dirección. Pero además, en un contexto de competitividad creciente, la participación en los resultados no se limita a una simple reducción de costos laborales, sino que procura mejorar la posición de la empresa, haciendo partícipes a los trabajadores en la elaboración e implementación de los cambios necesarios.

A diferencia de la participación en las utilidades, la participación en los resultados no puede ser decidida a nivel central, ya que solamente a nivel de la empresa es posible determinar los criterios más apropiados a su situación. Por lo tanto, las autoridades solamente pueden intervenir en forma indirecta, promoviendo la adopción de estos programas a través de incentivos fiscales, por ejemplo, o excluyéndolos de los pagos de las cargas sociales.
3 La presencia de un componente variable de ajuste automático
puede resultar insuficiente en el caso de una crisis aguda. Por ejem-
plo, en la industria automovilística argentina, donde una parte
significativa de las remuneraciones a los trabajadores era de ca-
rácter variable, tras la crisis asiática de 1997 se tomaron medidas más drásticas. Además de los recortes de costos operados por el mecanismo automático, se redujeron las horas extraordinarias, se aplicaron suspensiones y reducciones en las horas de trabajo y hubo programas de retiro voluntario y despidos (Soifer y Tomada, 1999). 


\section{III}

\section{Sistemas de participación en las utilidades de algunos países latinoamericanos y comparación con el sistema brasileño}

Como se dijo más atrás, varios países latinoamericanos cuentan con legislación que establece las formas de participación de los trabajadores en las utilidades de las empresas. Los sistemas presentan diferencias en diversos aspectos, que los llevan a tener efectos también diferentes.

En México, el sistema de participación en las utilidades usa como base la renta gravable de las empresas, $10 \%$ de la cual debe ser pagada a los trabajadores por ese concepto. ${ }^{4}$ Es importante señalar que las empresas no pueden deducir pérdidas de años anteriores para determinar las utilidades de las que participarán los trabajadores. A partir de 1988 se produjo una discrepancia entre la base para determinar la participación de los trabajadores en las utilidades y la base usada para el impuesto a la renta, debido a un ajuste por inflación que se aplica únicamente en este último caso. Tal diferencia es responsable de que la participación efectiva de los trabajadores sea menor que la originalmente prevista en la legislación.

La forma de distribución también está establecida por ley. La mitad de las utilidades en las que participan los trabajadores se distribuye en proporción a los salarios de cada trabajador (siendo su objetivo incentivar al personal de salario más alto) y el resto se distribuye en montos idénticos entre todos los trabajadores (siendo el objetivo redistribuir ingresos, ya que en términos relativos estos montos son más importantes para los trabajadores de salarios más bajos). Están exceptuadas de dar esta participación las empresas nuevas durante el primer año, las empresas nuevas de la industria extractiva durante el período de exploración, las instituciones sin fines de lucro y las empresas que facturan menos de 6 millones de pesos en el año.

En Chile se utiliza el lucro líquido como base para determinar la participación de los trabajadores en las utilidades. ${ }^{5}$ La utilidad líquida resulta de deducir del

\footnotetext{
${ }^{4}$ México, Ley Federal del Trabajo, Título $3^{\circ}$, capítulo VIII, artículos 117-131.

5 Chile, Código del Trabajo, Capítulo V: de las remuneraciones.
}

monto declarado para el impuesto a la renta, sin deducir las pérdidas de ejercicios anteriores, el $10 \%$ por concepto de remuneración al capital propio del empleador. El ámbito de aplicación está limitado a las empresas que legalmente están obligadas a presentar libros contables.

Pese a fijar en $30 \%$ el porcentaje por distribuir, el Código del Trabajo chileno establece una alternativa que libera a la empresa de la obligación anterior: la de pagar a los trabajadores una proporción del salario anual (25\%), con un límite máximo de 4.75 salarios mínimos por trabajador. Esta alternativa permite a las empresas de lucro alto restringir lo distribuido por participación, con un límite máximo que también resulta bastante bajo (cualquier trabajador con salario superior a 1.60 salarios mínimos estaría recibiendo menos del $25 \%$ de su salario anual). La forma de distribución es proporcional al salario de los trabajadores.

En la práctica, la modalidad predominante para el pago de la participación en las utilidades consiste en la proporción del salario anual con límite superior. Según una encuesta, esta modalidad se da en el $76 \%$ de los casos, mientras que el $30 \%$ de las utilidades se paga sólo en el $10 \%$ de los casos (Espinosa y Damianovic, 1999).

La legislación de Perú se diferencia de la de México y Chile en que las pérdidas de años anteriores pueden ser compensadas ${ }^{6}$ (Perú, Gobierno, 1996), y además establece diferentes niveles porcentuales de participación según la actividad de las empresas. Así, en las empresas mineras y comerciales y en los restaurantes la participación es del $8 \%$ de las utilidades; en las pesqueras, industriales y de telecomunicaciones es del $10 \%$, y en el resto del 5\%. En este país también existe un tope para la participación en las utilidades, pero éste es bastante alto (18 salarios mensuales). Al igual que en México, la distribución se hace $50 \%$ pro rata (igual para todos los trabajadores) y $50 \%$ en fun-

\footnotetext{
6 Perú, Gobierno (1996).
} 
ción del salario. Están exceptuadas de su aplicación las cooperativas, las instituciones sin fines de lucro y las empresas con 20 trabajadores o menos.

A diferencia del resto de los países mencionados, el sistema de participación en los beneficios aplicado en Venezuela tiene un mínimo y un máximo. ${ }^{7}$ El mínimo, equivalente a 15 días de salario, es aplicable a todas las empresas, incluso a las instituciones sin fines de lucro y las exentas de dar participación en los beneficios (aunque parezca contradictorio). Por otro lado, se fija un máximo diferenciado para las empresas con 50 trabajadores o más (cuatro meses de salario) y aquéllas con menos de 50 trabajadores (dos meses).

En este caso, la participación en los beneficios, concepto que implica pagos variables, en la práctica se transformó en un costo fijo más, dependiendo el valor de la participación del sector en que se desempeña la empresa. La introducción explícita de un mínimo y un máximo ciertamente ha contribuido a este resultado. Además, la magnitud de estos pagos es muy significativa ya que si bien el mínimo representa sólo el $4.16 \%$ del salario anual, el máximo equivale a $16.7 \%$ para empresas de menos de 50 trabajadores y a $33 \%$ para empresas con más de 50 trabajadores.

Entre los sistemas latinoamericanos de participación en las utilidades (gráfico 1) el mexicano aparece como el más "puro", ya que los pagos realizados por concepto de participación en los beneficios varían con el lucro logrado por la empresa. El establecimiento de un máximo en el caso de Chile busca restringir la participación de los trabajadores en aquellas empresas y sectores donde el lucro es muy alto (esto es menos evidente en el caso de Perú, donde el máximo parece ser bastante amplio). En vez de limitar la participación en estos sectores a través de un porcentaje menor, en Chile se prefiere limitarla a través de cierto número de salarios mínimos. En el otro extremo, Venezuela, con el establecimiento de un mínimo y un máximo, ilustra la desnaturalización de un instrumento que debería estar ligado al beneficio de la empresa: las características propias de la legislación llevan a aplicarlo como un pago fijo, que es mayor o menor - dentro del rango establecido-, dependiendo de la capacidad de pago general de la empresa, pero que por lo general no es variable.

En tanto que el sistema de participación en las utilidades predomina en América Latina, Brasil presen-

\footnotetext{
7 Venezuela, Ley Orgánica del Trabajo, Capítulo III: de la partici-
} pación en los beneficios, artículos 174-184.
GRAFICO 1

\section{Sistemas de participación en las utilidades}

Sistemas "puros"
Sistemas con "techo" $\begin{aligned} & \text { Chile (4.75 salarios mínimos) } \\ & \text { Perú (18 salarios) } \\ & \begin{array}{l}\text { Sistemas con límite } \\ \text { inferior y superior }\end{array}\end{aligned}$

ta la opción de adoptar sistemas de participación en los resultados. Aunque en casi todos los países analizados existen empresas, especialmente del sector moderno, que cuentan con programas de participación en los resultados, ${ }^{8}$ Brasil es el único país de la región en que la legislación ofrece la posibilidad de optar entre estas dos alternativas, que disfrutan de los mismos incentivos en términos de exenciones de contribuciones empresariales.

Si se compara el sistema de Brasil con los demás sistemas latinoamericanos expuestos, vemos en primer lugar que el sistema brasileño no está ligado únicamente a las utilidades, ya que puede optarse por vincular la participación a resultados. Aunque es posible regular la participación en las ganancias (por lo general, fijando porcentajes), la participación en los resultados no puede ser reglamentada por la autoridad central, ya que se encuentra eminentemente en el dominio de la empresa (aunque en algunos casos las partes han llegado a acuerdos colectivos por ramas o sectores).

Otra diferencia, menos importante, es que en Brasil no se establecen formas de distribución entre los trabajadores, precisamente para dar mayor libertad en el caso de que una empresa elija la participación en los resultados. Por ejemplo, si se toma como referente el resultado de un grupo de trabajo y se quiere potenciar la solidaridad entre los miembros del grupo, se puede preferir realizar pagos iguales a todos los trabajadores del mismo. Pero si se quiere privilegiar la productividad individual, la forma de distribución más apropiada dependerá del salario de cada uno de ellos. Estos problemas no se presentan cuando se trata de distribuir la participación en los lucros.

\footnotetext{
8 Por ejemplo, un estudio de la CEPAL resume una serie de casos de "salarios participativos" en empresas chilenas grandes, en las cuales la parte variable de los salarios constituye un porcentaje significativo de la remuneración total. El mismo estudio incluye algunos ejemplos de empresas brasileñas que aplicaban programas de participación con anterioridad a la introducción de la MP 794 (CEPAL, 1992).
} 
El sistema brasileño, además, sólo exceptúa expresamente a las instituciones sin fines de lucro, y por esto se puede caracterizar como de aplicación universal por su amplitud. Como en la MP se establece que todas las empresas deben negociar la participación de los trabajadores, ya sea en los lucros o en los resultados, es posible abarcar empresas muy diversas -menores, mayores; más nuevas, más antiguas; con mayor o menor participación en el mercado; con mayor o menor lucro- ya que en cada una se tomarán en cuenta sus particularidades.
Este último aspecto es probablemente el más distintivo: el sistema brasileño coloca en primer plano la necesidad de negociación entre las partes, en tanto que los otros sistemas establecen una forma de cálculo y aplicación muy clara, que no requiere de mayores discusiones. En este sentido, la diferencia con el caso chileno es muy evidente, ya que la opción de elegir entre dar participación en un porcentaje de las ganancias o efectuar un pago limitado en función del salario es prerrogativa exclusiva del empleador, quien normalmente elige la opción más barata para su empresa.

\section{IV}

\section{Evaluación de un sistema "puro" de participación en las utilidades: el caso de México}

Como todo instrumento legal, éste solamente puede ser evaluado a partir de su implementación. Seguidamente se presentarán algunos criterios utilizados en 1996 en México por una comisión tripartita encargada de revisar el porcentaje de participación de los trabajadores en las utilidades de las empresas (PTU). ${ }^{9}$ En México, cada 10 años se debe reunir una comisión con el único objetivo de ajustar el porcentaje de participación. Para ello se establece una secretaría, que prepara estudios basados en datos existentes o estadísticas específicamente recogidas, y analiza la respuesta de los actores sociales a cuestionarios que apuntan a conocer la opinión de las organizaciones de trabajadores y de empresarios. El objetivo de esta comisión parece ser bastante limitado, ya que no puede sugerir cambios o mejoras en el instrumento legal ni en los mecanismos de aplicación. De todas formas, los criterios utilizados por la comisión pueden servir de fuente de información y de reflexión.

Según datos de la Secretaría de Hacienda sobre el lucro fiscal y la participación de los trabajadores en los beneficios que han de ser distribuidos, aunque la ley dispone que como mínimo se debe distribuir el 10\% de los beneficios, en los años noventa nunca se alcanzó tal porcentaje. El año en que se llegó a un valor más próximo fue 1990, cuando se distribuyó el 8.2\% (gráfico 2).

9 Las informaciones presentadas en esta sección se basan en las Memorias de dicha comisión.
GRAFICO 2 México: Participación de los
trabajadores en las utilidades, 1990-1994
(Como porcentaje del beneficio fiscal total)

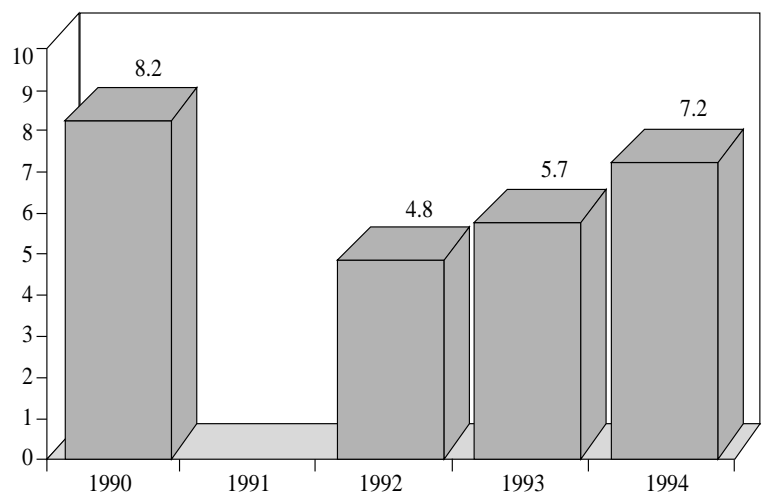

Una parte de la diferencia entre lo determinado por ley y la participación efectivamente distribuida se explica por una divergencia contable entre la base que se utiliza para determinar el pago por impuesto a la renta, que es ajustada por inflación, y la base para definir la participación de los trabajadores, que no lleva tal ajuste. Otra parte de la diferencia se explica por una subestimación de la PTU. Esta información demostraría que, en la práctica, no se estaría distribuyendo el mínimo de $10 \%$ de las utilidades.

Informaciones del Instituto de Estadística muestran que las utilidades por distribuir son muy diferentes de un sector de la economía a otro. La mayor parte de la PTU de México se distribuyó siempre en el sector 
industrial: el 64\% de ella en 1995 y el 69\% en 1993. En el sector comercial la PTU cayó apreciablemente en esos años, pasando de 24 a $17 \%$ de la PTU total, mientras que en el sector servicios mostró un pequeño aumento (de 12 a $15 \%$ ).

Sin embargo, esta información no es la más pertinente, ya que el sector industrial también es el mayor empleador del segmento formal. Por lo tanto, un indicador más apropiado sería el de las utilidades distribuidas por trabajador ocupado, es decir, por trabajador del segmento formal al que concierne la declaración de utilidades de las empresas. En 1994, el número de trabajadores en esa situación era de 10.7 millones, en una población económicamente activa un poco superior a 35 millones.

El sector industrial siempre distribuyó una cantidad mayor por trabajador ocupado que el resto de los sectores económicos (cuadro 1). En años recientes, esa diferencia fue aumentando: mientras en 1985 los pesos distribuidos por la industria eran 1.5 veces más que los distribuidos por el comercio, en 1993 fueron 3.5 veces más. También hubo convergencia entre el comercio y los servicios, producida fundamentalmente por la caída de las utilidades distribuidas por trabajador en el sector comercial.

¿Cuánto representan esos pesos distribuidos por trabajador como porcentaje de la remuneración total en cada uno de los sectores? En 1985, por ejemplo, el lucro distribuido por trabajador en la industria fue de 690 pesos (50\% mayor que el distribuido por el comercio y 2.4 veces la cantidad distribuida por los servicios). Pero en términos de la remuneración total de los trabajadores en esos mismos sectores, la importancia relativa de lo distribuido por concepto de PTU en la industria era menor que en el comercio y casi idéntica al porcentaje del sector servicios, sencillamente porque el salario medio en la industria era mucho más alto que en los otros dos sectores.

CUADRO

\section{México: Utilidades distribuidas por trabajador ocupado (Pesos de 1994)}

\begin{tabular}{lrrr}
\hline Sectores & 1985 & 1988 & 1993 \\
\hline Industria & 690.2 & 812.2 & 713.1 \\
Comercio & 463.2 & 349.9 & 204.1 \\
Servicios & 293.0 & 211.9 & 207.2 \\
Industria/comercio & 1.5 & 2.3 & 3.5 \\
Industria/servicios & 2.4 & 3.8 & 3.4 \\
\hline
\end{tabular}

Fuente: México, Gobierno (1996).
Sin embargo, para evaluar el impacto real de un sistema de participación en los lucros o en los resultados, no sólo es importante conocer las utilidades distribuidas por trabajador ocupado, sino también saber qué proporción son de la remuneración total o del salario base. En 1993, por ejemplo, si bien la cantidad distribuida por trabajador en el sector comercio era prácticamente igual a la del sector servicios, la proporción de la remuneración total distribuida fue más importante en el comercio, donde representó $2.1 \%$ de la remuneración total (cuadro 2).

Los porcentajes que muestra dicho cuadro, entre 1.5 y $4 \%$, pueden dar la idea de una distribución muy baja. Sin embargo, cabe señalar, por un lado, que estos promedios encubren grandes diferencias dentro de cada uno de los sectores, industrias y empresas: en la industria manufacturera, los trabajadores de productos alimenticios y químicos recibieron una compensación por PTU mayor que el promedio de las industrias en los tres años analizados, mientras que los trabajadores de la madera y los textiles estuvieron por debajo del promedio (cuadro 3). Y por otro lado, que los promedios se vieron disminuidos por la inclusión de trabajadores

CUADRO 2

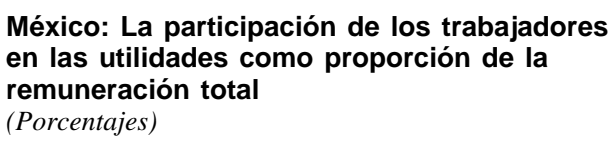

\begin{tabular}{lccc}
\hline Sector & 1985 & 1988 & 1993 \\
\hline Industria & 2.8 & 4.0 & 3.2 \\
Comercio & 4.2 & 4.3 & 2.1 \\
Servicios & 2.4 & 2.3 & 1.5 \\
Total nacional & 3.0 & 3.8 & 2.5 \\
\hline
\end{tabular}

Fuente: México, Gobierno (1996).

CUADRO 3 México: La participación de los
trabajadores en las utilidades como
proporción de la remuneración total
(Porcentajes)

\begin{tabular}{lccc}
\hline Industria & 1984 & 1988 & 1991 \\
\hline Ind. manufacturera & 2.6 & 5.4 & 4.9 \\
Prod. alimenticios & 3.5 & 5.5 & 5.2 \\
Textiles & 2.6 & 2.6 & 1.6 \\
Papel & 2.6 & 9.0 & 7.8 \\
Prod. químicos y & & & \\
$\quad$ derivados del petróleo & 4.3 & 8.0 & 6.2 \\
Metales básicos & 1.1 & 3.1 & 3.4 \\
Madera & 1.3 & 2.1 & 2.8 \\
\hline
\end{tabular}

Fuente: México, Gobierno (1996). 
que no recibieron nada por concepto de PTU, o montos muy pequeños, debido a que sus empresas no generaron utilidades. Por lo tanto, en sectores manufactureros como los de alimentos, papel, productos químicos y derivados del petróleo, los montos pagados en las empresas que tienen utilidades resultan significativos, ya que se pueden estimar como superiores al $10 \%$ de la remuneración anual.

\section{V}

\section{Evaluación preliminar del sistema brasileño}

Pese a que la Medida Provisional 794 no constituye la ley definitiva, ella viene siendo aplicada desde hace cinco años. Por lo tanto, existe ya una práctica de participación de los trabajadores en los lucros y resultados (PLR) que permite hacer una evaluación preliminar de los aspectos positivos y negativos de la MP, así como de la eficacia de sus estipulaciones.

Para evaluar adecuadamente el sistema brasileño es preciso, en primer lugar, saber en qué medida se cumple con las pocas disposiciones que la MP establece como obligatorias, para lo cual se necesita información macroeconómica sobre la situación y, en menor medida, información acerca de lo que ocurre en las empresas mismas.

En segundo lugar, hay que saber qué sucede con los aspectos abiertos a negociación y si los resultados son los que deseaban los legisladores, sindicatos y empleadores, así como las autoridades. Aunque para este fin es posible utilizar algunos datos agregados, lo fundamental es conocer lo que está aconteciendo a nivel de la empresa.

\section{Cumplimiento de disposiciones obligatorias}

Para verificar el cumplimiento de estas disposiciones es importante conocer el número de empresas (y el número de trabajadores) que negociaron con éxito la participación en los lucros o resultados; el número de empresas en que la negociación llegó a un punto muerto (y, entre ellas, los casos en que se está en proceso de mediación y arbitraje) y el número de empresas que aún no han negociado.

Esta información, comparada con el universo potencial, indicará si se está cumpliendo con la disposición de negociar establecida en el artículo 2 de la Medida Provisional. ${ }^{10}$ Esto es de particular importan-

\footnotetext{
${ }^{10}$ Para los fines de esta Medida Provisional las personas físicas y las entidades sin fines de lucro, únicas excepciones que se establecen a la negociación entre la empresa y sus empleados, no son equiparadas a la empresa.
}

cia porque en dicha MP no se establecen plazos para la negociación, ni hay sanciones previstas si una de las partes rehúsa negociar. A partir de la información antedicha, puede determinarse si esos problemas se dan en especial en alguna región, en algún sector económico o en empresas de algún tipo clasificadas, por ejemplo, según su tamaño.

Lamentablemente, no existe hasta el momento una fuente que procese todos los acuerdos firmados. Sin embargo, no es aventurado pensar que la aplicación de la participación en los lucros y en los resultados es aún muy limitada fuera de las empresas grandes y medianas de la industria manufacturera del sudeste brasileño.

Otra disposición de cumplimiento obligatorio está ligada a la frecuencia del pago de la participación de los trabajadores. Se debe recordar que la Medida Provisional fija un período mínimo de seis meses. Al respecto, hay que observar si la frecuencia mínima está siendo respetada o si se prefiere la distribución anual, y determinar si la distribución se concentra en alguna época del año, lo que puede tener importancia económica por su efecto sobre el consumo.

En el caso brasileño, desde un comienzo existió cierta preocupación de las autoridades por evitar que los pagos por participación en los lucros o los resultados fueran utilizados para desviar pagos que deberían ser por salario base (beneficiándose así de la exención de contribuciones sociales). En la MP, esta preocupación se tradujo en el establecimiento de una periodicidad mínima de seis meses para el pago de la participación, suponiendo que es imposible postergar un desvío del salario base por un período tan largo. En el marco de amplia libertad de negociación que establece la Medida Provisional esta disposición es una de las pocas de cumplimiento obligatorio.

Sin embargo, del análisis de algunos acuerdos de participación en los lucros o los resultados se desprende que tal disposición no está siendo respetada en todos los casos, ya que en algunos acuerdos se establece una periodicidad inferior a los seis meses. Según un estu- 
dio realizado por el Departamento Intersindical de Estadística y Estudios Socioeconómicos, basado en 448 acuerdos firmados en 1996, un 6\% de ellos establecían pagos en tres cuotas, mientras que el $2 \%$ estipulaban más de tres pagos por año (DIEESE, 1996). El Profesor Hélio Zylberstajn, en una revisión no sistemática de acuerdos de participación en lucros o resultados, encontró que cerca del $20 \%$ de ellos tenía una frecuencia de distribución inferior a los seis meses $(22 \%$ en 1995 y $17 \%$ en 1996). ${ }^{11}$ Además, la práctica de otorgar anticipos de esa participación no es inusual, lo cual significa pagos más frecuentes que lo estipulado por la MP 794. De generalizarse esta forma de aplicación, se estaría generando un desfinanciamiento de los servicios sociales, así como una "desalarización" de la economía. Así, este instrumento puede llegar a debilitar el frente fiscal ${ }^{12}$ y distorsionar las estructuras salariales.

Parecería, entonces, que éste es uno de los aspectos que debe ser fiscalizado, en tanto continúe vigente esa disposición. Otra opción es establecer un límite máximo (en forma de porcentaje del salario base) al monto pasible de exención de pago de cargas sociales y previsionales, liberando la frecuencia de su distribución a lo acordado en cada empresa.

En cuanto al posible impacto de que los pagos por participación en el lucro o en los resultados se concentren en algún período del año, tampoco se cuenta con información. Según la muestra del DIEESE, en 1996 se habrían distribuido cerca de 750 millones de reales. Este dato refleja que, de extenderse su aplicación, la participación en el lucro o en los resultados puede representar un volumen tal que pase a constituir un factor importante a nivel macroeconómico.

\section{Las negociaciones en Brasil}

Puesto que en Brasil la adopción de programas de participación en el lucro o en los resultados se da por negociación entre las partes, interesa conocer quiénes

\footnotetext{
${ }^{11}$ Esta información fue presentada por el Profesor Zylberstajn durante el seminario "Experiencias de participación de los trabajadores en los lucros o resultados de las empresas", que tuvo lugar en São Paulo el 14 de agosto de 1997. Las menciones que se hacen más adelante a Zylbenstajn se refieren a sus intervenciones en este Seminario.

${ }^{12}$ Esta afirmación cobra una dimensión mayor cuando se observa que las empresas que aplican programas de participación en los lucros o en los resultados son, por lo general, empresas grandes del sector manufacturero que están, al mismo tiempo, entre los mayores contribuyentes. Si este grupo disminuye sus contribuciones, el impacto fiscal puede ser muy significativo.
}

realizan la negociación. En un comienzo, la Medida Provisional dejaba la representación de los trabajadores en el proceso de negociación a cargo de la comisión de empleados que se eligiera con ese propósito. Sólo recientemente se dio un papel al sindicato mediante una modificación introducida por la MP 1539-34 en agosto de $1997 .{ }^{13}$ Pese a ese vacío inicial, es interesante destacar que las negociaciones que han tenido lugar han involucrado a los sindicatos en la mayor parte de los casos. Además, en varias ocasiones fueron los sindicatos los que promovieron la convocación a negociaciones respecto a esta participación, que incluso llegó a ser motivo de huelgas.

Zylberstajn encontró que en los acuerdos firmados en 1996 la contraparte de las empresas fue el sindicato en el $29 \%$ de los casos y el sindicato y la comisión de empleados en el 34\% (gráfico 3). Por lo tanto, las comisiones de empleados negociaron y firmaron los acuerdos en representación de los trabajadores únicamente en una tercera parte de los casos analizados.

También es aconsejable establecer cuál fue el criterio escogido por las partes en la negociación: si fue el lucro o los resultados (y cuál fue el preferido), o simplemente la distribución de un monto fijo pagado por concepto de participación de los trabajadores en el lucro, pero sin relación alguna ni con las ganancias de la empresa ni con la obtención de algún resultado específico. Por más que esta última opción no esté incluida entre las que ofrece la Medida Provisional (y además esté alejada de su espíritu), bien puede ser el resultado de la negociación de las partes en una empresa o sector.

El objetivo de recabar esta información es tener una idea acerca de cuál puede ser el impacto de estas

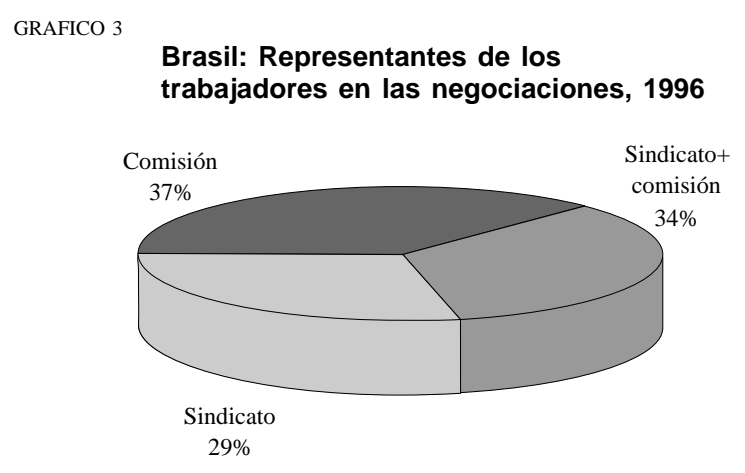

\footnotetext{
13 "La participación en los lucros o resultados será objeto de negociación entre la empresa y sus empleados, mediante comisión elegida por éstos, integrada además por un representante indicado por el sindicato de la categoría respectiva" (artículo $2^{\circ}$ ).
} 
formas de participación sobre la economía. Si se elige el lucro como criterio primordial, se privilegia la idea de distribución y la participación de los trabajadores fluctuará con el desempeño financiero de la empresa. Si se opta por los resultados, probablemente se está buscando modificar algún comportamiento y apoyar de esa forma la introducción de mejoras. Finalmente, si se elige la distribución de un monto fijo simplemente para cumplir con la MP sin tomarse el trabajo de elaborar un programa de objetivos, se está aumentando el costo laboral sin que la empresa obtenga nada en particular a cambio: ni se apoya un cambio o intento de mejora, ni se obtiene la flexibilidad que da un componente variable, como en el caso de la participación ligada al lucro. Por lo tanto, de las tres alternativas el criterio de resultados es el que puede tener efectos repetitivos, ya que los parámetros pueden ser reemplazados o ajustados con el tiempo, mejorando aún más los logros del trabajo y, por ende, la competitividad de la empresa. Por el contrario, el efecto de aplicar el criterio de lucro o de un pago fijo se hará sentir principalmente a nivel macroeconómico al momento de su introducción, a través de un aumento del consumo, pero no mejorará la competitividad de la empresa.

Cuando se dictó la MP, por el lado empresarial se tendió a mirar la participación en el lucro o en los resultados como un costo laboral adicional. Había, además, un desconocimiento general sobre qué factores podían constituir resultados y cómo se podrían utilizar, de modo que no se percibía el potencial de este instrumento como herramienta de gestión. Por otro lado, se entendía que una vez otorgada una retribución por concepto de participación en el lucro o en los resultados, ésta debería ser mantenida como base en los ejercicios siguientes, lo cual no es así ya que se trata de un componente variable de la remuneración. La lista de dudas no estaría completa si no se mencionara la desconfianza mutua de las partes, respecto al acceso a información que la dirección considera prerrogativa empresarial (por el lado de la empresa), y respecto a la calidad de la información, en especial de la financiera (por el lado de los sindicatos).

En cambio, lo que quedó en claro desde el principio fue que las retribuciones por concepto de participación en el lucro o en los resultados estarían exentas de las contribuciones sociales. Esto alentó al lado empresarial a adoptar los primeros programas de esta índole. En una primera etapa, la participación en el lucro o en los resultados se aplicó en aquellas empresas o sectores con mayor organización sindical y, en la mayoría de los casos, los pagos por este concepto consistieron en montos fijos no relacionados con la obtención de lucro ni el logro de algún resultado previamente convenido. Si bien esta forma de aplicación no se ajusta al espíritu de la MP, se la considera válida por ser el resultado de la negociación entre las partes. Según una muestra realizada por el Departamento Intersindical de Estadística y Estudios, apenas el 12.6\% de los acuerdos analizados incluía algún tipo de metas (DIEESE, 1996).

En una segunda instancia, se avanzó hacia negociaciones basadas en la fijación de metas, con la escala de retribuciones correspondientes. En su estudio, Zylberstajn identificó una disminución en el número de acuerdos no condicionados al logro de metas. ${ }^{14} \mathrm{Se}$ puede decir entonces que la utilización del instrumento de participación habría dado un salto cualitativo, al constituirse en un elemento que puede contribuir a elevar el nivel de la empresa y, por ende, a que haya un "financiamiento genuino" del pago por participación en el lucro y en los resultados.

En una tercera etapa, es preciso conocer la cantidad distribuida como porcentaje de la remuneración total de los trabajadores, para verificar si existen diferencias importantes entre empresas de distintos sectores o tamaños y observar si este componente nuevo de la remuneración va ganando importancia o si su magnitud es superior a la "deseada".

El monto de los pagos realizados por concepto de participación en el lucro y en los resultados es, en promedio, muy significativo en comparación con el

GRAFICO 4

Brasil: Participación en el lucro o en los resultados, por tamaño de la empresa

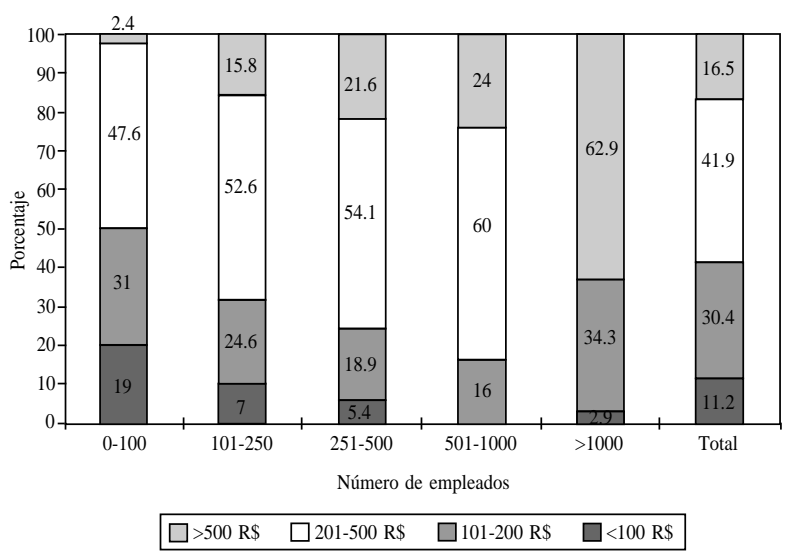

14 El estudio no abarcó el mismo grupo de empresas en ambos años, por lo cual las tendencias señaladas constituyen una aproximación a lo sucedido. 
salario base. Una encuesta realizada por la consultora Mercer Mw, enfocada hacia empresas grandes de capital tanto nacional como extranjero, estableció que, en promedio, los pagos por este concepto en 1996 fueron equivalentes a 1.16 salarios por empleado, y que los pagos de las empresas que daban un monto fijo fueron, en promedio, de casi 1000 reales (aproximadamente 1000 dólares). Cabe señalar, sin embargo, que en la magnitud de los pagos por participación en el lucro o en los resultados influye el tamaño de la em-

\section{VI}

\section{Conclusiones}

¿Cuáles son los aspectos de los sistemas de participación en las utilidades y en los resultados que mejor se adaptan al contexto económico en que se desempeñan las empresas y cuáles son los obstáculos que se presentan? Por el lado de los sistemas "puros" de participación en las utilidades, el aspecto que mejor se adapta a las nuevas exigencias es la flexibilidad a la baja de este costo, lo cual cuadra con esquemas de tipo "defensivo" de corto plazo. ${ }^{15}$ Ante una reducción en la demanda que afecte a las utilidades, la primera reacción de las empresas es ajustar los costos. En este caso, el componente de participación en las utilidades se ajusta en forma automática.

La flexibilidad de los sistemas de participación en los resultados, por su parte, no origina en sentido estricto una baja de los costos absolutos (aunque sí puede resultar en una caída de los costos unitarios); de hecho, puede manifestarse de otras formas. Por ejemplo, un programa que mejore la calidad del producto tiene un impacto indirecto sobre los costos de producción, al evitar la necesidad de rehacer piezas, al disminuir las devoluciones, al provocar menos quejas de clientes, etc. Como las formas que tome el programa se adaptarán a las necesidades estratégicas propias de la empresa, no se trata de un planteamiento defensivo sino más bien de uno estratégico de mediano y largo plazo.

¿En qué medida estos distintos enfoques se ajustan a la nueva realidad del contexto internacional? $\mathrm{Si}$

\footnotetext{
${ }^{15}$ Esta flexibilidad no se presenta, por ejemplo, en el caso de Venezuela, donde hay un pago mínimo y donde la práctica ha convertido los pagos por este concepto en bonos fijos.
}

presa (gráfico 4). La muestra del DIEESE, que abarca un espectro más amplio de empresas que la encuesta de Mercer, indica esto con claridad.

Que la magnitud de los pagos realizados sea grande es, por un lado, muy positivo ya que constituye un verdadero incentivo para los trabajadores. Pero, por otro lado, puede estar indicando que en algunos casos se está desviando lo que deberían ser pagos de salario base hacia pagos flexibles por concepto de participación, que no conllevan contribuciones sociales.

bien el enfoque defensivo resulta importante en períodos de crisis, las exigencias que impone en cada empresa la mayor competitividad a nivel internacional hacen que el planteamiento estratégico resulte más importante en el largo plazo. En este sentido, la participación en los resultados puede constituir un instrumento adecuado para acelerar la reestructuración necesaria de muchas empresas, ya que da la posibilidad de conjugar la consecución de un objetivo estratégico de la empresa con el incentivo a los trabajadores necesario para alcanzarlo.

Sin embargo, no hay que minimizar las dificultades que estos programas encierran. Es más difícil identificar los cuellos de botella en una empresa y definir las formas de resolverlos que aplicar un sistema automático de reducción parcial de costos en épocas de crisis. Los sistemas de participación en los resultados resultan bastante complejos. Se debe poner sumo cuidado tanto en la identificación del factor que habrá de ser remunerado como en el establecimiento del parámetro de medición/evaluación y en las formas de distribución entre los trabajadores y su implementación. Por lo general, se trata de procesos largos y su seguimiento debe ser muy afinado.

Por lo dicho, salta a la vista la ventaja de la simplicidad de los sistemas de participación en las utilidades que establece la legislación en América Latina. Su carácter obligatorio y la aplicación genérica de un mismo coeficiente hacen de su aplicación un proceso extremadamente sencillo. Sin embargo se ha comprobado que en la práctica no siempre se cumple estrictamente con lo establecido por la ley, lo cual pone en tela de juicio la eficacia de estos sistemas como instrumento de distribución de ingresos. 
Pero además, la aplicación de un coeficiente único de participación en las utilidades para empresas de muy diversas características no parece ser suficientemente flexible. Por ejemplo, distribuir $10 \%$ de las utilidades por concepto de participación no significa lo mismo para una empresa dominante en su mercado que para otra con un mercado atomizado, ni tampoco es igual para una empresa que está en fase inicial de crecimiento que para otra consolidada en su sector. La imposibilidad de adaptar el instrumento a la realidad de la empresa limita fuertemente su utilización como sistema de incentivos, ya que se asemeja más a un derecho adquirido de los trabajadores.

¿Qué tipo de empresas se podría beneficiar mayormente de la aplicación de programas de participación en los resultados? Muchas empresas grandes y muchas de las transnacionales a nivel regional ya aplican alguna de las formas de participación en los resultados, aunque la legislación nacional no las promueva, y utilizan estos programas como un instrumento de gestión y de mejora en las relaciones laborales. Sin embargo, este tipo de instrumentos continúa siendo bastante desconocido entre las empresas medianas y pequeñas. A este nivel, la introducción de un instrumento legal que promueva la adopción voluntaria de sistemas de participación en los resultados (con algún beneficio fiscal) podría difundir tal alternativa.

No hay que pensar que con sólo promover la participación en los resultados los sistemas que se adopten necesariamente perseguirán el logro de mejoras. El ejemplo de Brasil muestra que, ante la dificultad de aplicar sistemas basados en resultados, muchas empresas comenzaron a distribuir montos adicionales en forma de bonos por ese concepto, aunque sin condicionarlos al logro de algún resultado. Pero esta actitud inicial fue siendo paulatinamente modificada al irse comprendiendo mejor el potencial de los sistemas de participación. Vemos así que éste no es un instrumento de "aplicación perfecta inmediata", sino uno para cuya aplicación efectiva se necesita un período de aprendizaje.

Los sistemas de participación en las utilidades y los de participación en los resultados también presentan diferencias en materia de relaciones laborales y de políticas de recursos humanos. Los sistemas de participación en las utilidades no precisan de una gran participación de los trabajadores o de los sindicatos. Cuando se trata de programas elaborados a nivel de la empresa, la participación se puede dar en la negociación del porcentaje, la forma de distribución y otros aspectos, para lo cual bastará con una reducida representación de los trabajadores. En los sistemas predominantes en América Latina, donde la legislación determina las condiciones de la participación en las utilidades, la participación de los trabajadores es nula, o limitada sólo a un papel contralor. Esta forma de regulación parece ser propia de una visión paternalista del Estado.

Los programas de participación en los resultados, por el contrario, precisan de la participación directa de los trabajadores mismos así como del sindicato en diversas instancias del proceso (desde la identificación de las variables estratégicas hasta el seguimiento de su aplicación). En este sentido, las estipulaciones de la MP brasileña resultan apropiadas ya que llaman a las partes a negociar y a convenir el tipo de programa más adecuado en cada caso. La imperiosa necesidad de diálogo entre las partes y de transparencia en el proceso hacen de estos programas un valioso instrumento para establecer políticas de recursos humanos más participativas y relaciones laborales no conflictivas, que concuerden más con la necesidad de mejorar la competitividad de las empresas.

\section{Bibliografía}

Card, D. (1990): Comment to Weitzman and Kruse, A. Blinder (ed.), Paying for Productivity. A Look at the Evidence, Washington, D.C, The Brookings Institution.

CEPAL (Comisión Económica para América Latina y el Caribe) (1992): Equidad y transformación productiva: un enfoque integrado, LC/G.170/Rev.1-P, Santiago de Chile.

DIEESE (Departamento Intersindical de Estadística y Estudios Socioeconómicos) (1996): Participação nos lucros e resultados: um painel das negociações, Boletim DIEESE, $\mathrm{N}^{\circ} 184$, São Paulo.

Espinosa, M. y N. Damianovic (1999): Encuesta laboral 1998. Informe de resultados, Santiago de Chile, Dirección del Trabajo de Chile, Departamento de Estudios.
Marinakis, A.E. (1997): A participação dos trabalhadores nos lucros e resultados no Brasil: um instrumento para acelerar a restructuração necessária; Revista de Administração de Empresas, vol. $37, \mathrm{~N}^{\circ} 4$, São Paulo.

México, Gobierno (1996): Memoria de labores, México D.F., Cuarta Comisión Nacional para la Participación de los Trabajadores en las Utilidades de las Empresas.

Perú, Gobierno (1996): Participación de los trabajadores en las utilidades de las empresas generadoras de rentas de tercera categoría, Decreto legislativo, $\mathrm{N}^{\circ} 892$, Lima, 8 de noviembre.

Perry, C. y D. Kegley (1990): Employee Financial Participation: An International Survey, Multinational Industrial Relations Se- 
ries, $\mathrm{N}^{\circ} 12$, Philadelphia, The Wharton School, University of Pennsylvania,.

Soifer, R. y C. Tomada (1999): Impactos de la inversión extranjera sobre el mercado laboral y las relaciones laborales en la $\mathrm{Ar}$ gentina en los años 1990, informe preparado para la OIT.
Weitzman, M. (1984): The share economy, Cambridge, Massachusetts, The MIT Press.

Weitzman, M. y D. Kruse (1990): Profit sharing and productivity, A. Blinder (ed.), Paying for Productivity. A look at the Evidence, Washington D.C., The Brookings Institution. 